\title{
THE UNBUILT MUSMECI PARABOLIC CROSS VAULT REINVENTED AS A DRY-MASONRY STRUCTURE
}

\section{CLAUDIO INTRIGILA*, NICOLA A. NODARGI AND PAOLO BISEGNA}

\author{
Department of Civil Engineering and Computer Science (DICII) \\ University of Rome Tor Vergata \\ via del Politecnico 1, 00133 Rome, Italy \\ e-mail: \{intrigila*, nodargi, bisegna\}@ing.uniroma2.it, www.dicii.uniroma2.it (*corresponding \\ author)
}

Keywords: Cross Vault, Musmeci Vault, Dry-masonry Structure, Limit Analysis, Parabolic Masonry Arch, Spreading Supports

\begin{abstract}
This paper investigates the unbuilt Musmeci parabolic vault, reinventing the original reinforced concrete structure as a dry-masonry vault. In the framework of rigid no-tension constitutive model with no sliding, the equilibrium analysis is conducted with the aim of evaluating the design thickness of the masonry vault, respecting the original Musmeci shape. A parametric survey is performed to assess the minimum thickness of the vault, and its structural capacity under spreading supports. Attention is focused on the different collapse mechanisms and the corresponding crack patterns. For a better insight into the behaviour of the parabolic vault, the relevant case of the parabolic arch is first analysed and discussed. The numerical results show the feasibility of the project, with a thickness comparable with that proposed by Musmeci.
\end{abstract}

\section{INTRODUCTION}

In 1954 the Italian engineer Sergio Musmeci (1926-1981) conceived a reinforced concrete parabolic cross vault, with a triangular bay, featured by the assembly of three triangular webs. Designed for a small market in southern Italy through a prime structural optimization experiment, the unique vault remained unbuilt [1].

Despite the interesting geometric solution and the pioneering structural optimization procedure, the project remained forgotten and mentioned only in [8] as "exemplary for its modular conception of the structure based on the repetition of cylindrical shells".

In this framework, a novel perspective of the elegant geometry of the Musmeci vault is explored here, revisiting the original reinforced concrete structure. In particular, the possibility of reinventing the original vault as a dry-masonry structure is investigated, adopting the limit analysis theory [2-7].

Aim of this work is, on the one hand, to bring attention to the unique and forgotten geometry proposed by Musmeci, on the other hand, to analyse the structural behaviour of parabolic masonry vaults, still not be researched in detail.

In order to be as faithful as possible to the original geometry proposed by Musmeci, the only free parameter considered in the design process is the vault thickness. Therefore, different 
aspects are involved in the proposed procedure.

A first aspect is related to the discretization process of the original geometry as a dry assemblage of blocks, researching on the proper stereotomy of blocks and evaluating their influence on the static admissibility of the vault.

A second aspect is related to estimating a design thickness with a reasonable factor of safety. Accordingly, a set of parametric analyses is conducted to evaluate the influence of the vault thickness on the global response. In particular, the minimum admissible thickness and the minimum thrust of the vault are investigated. Furthermore, since the vault behaviour is deeply influenced by finite displacements at the springing $[3,5,7]$, the Musmeci vault is analysed under horizontal settlements.

According to the Heyman sliced model $[2,3,7]$, the vault is modelled as combination of web arches and ribs and their capacity on spreading supports is evaluated in terms of ultimate displacements and relative thrusts. In this context, attention is focused on the different collapse mechanisms and on the pertaining crack patterns.

The present paper is organized as follows. Section 2 is devoted to a brief review of the Musmeci structural model. The discretization process and the main assumptions of the proposed model are reported in Section 3. Since the behaviour of the parabolic square cross vault is closely connected to the behaviour of its diagonal ribs [7,9], the case of the parabolic arch on spreading supports is first analysed and discussed in Section 4. Then, the minimum thickness of the Musmeci vault and its displacement capacity are analysed in Section 5. Finally,

\section{conclusions are outlined in Section 6}

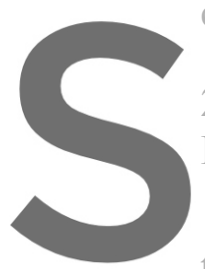

\section{THE MUSMECI MODEL: A SYSTEM PARABOLIC CROSS V \\ Designed in 1954 by Sergid
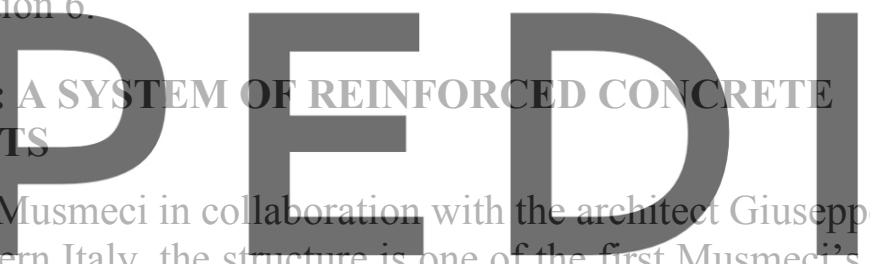

to cover a rural market in s

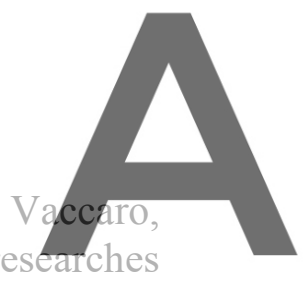

on the minimum structural design criteria $[1,8]$

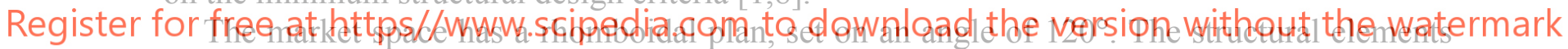

that compose the cover of the market are the same that determine its shape. It is, indeed, a modular structure consisting of the repetition of 4 tripartite vauits, each one with an equilateral triangular bay. Each vault is formed by three triangular webs with a parabolic profile. The external parabolic arches of the webs have a rise, $f$, equal to $3.65 \mathrm{~m}$ and a span, $2 a$, of $12.50 \mathrm{~m}$, as shown in Figure 1. The ribs, generated by the intersection of the 3 webs, are also semiparabolic arches and form angles of $120^{\circ}$ in plan.

(a)

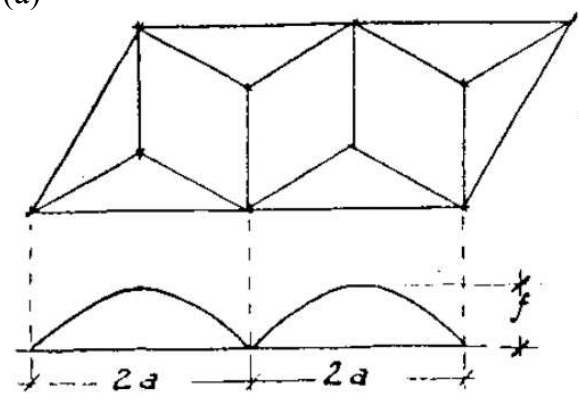

(b)

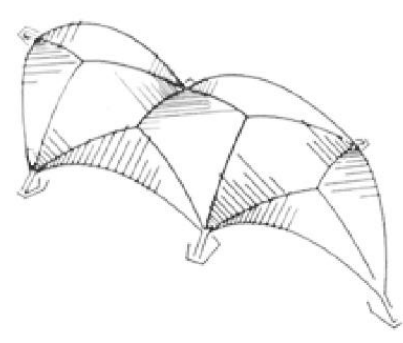

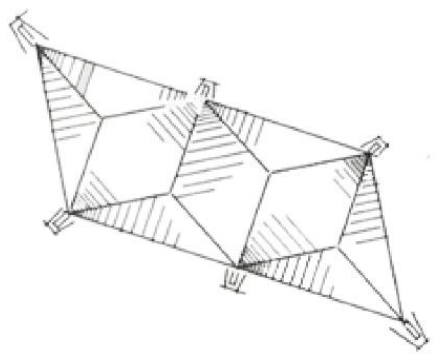

Figure 1: (a) Schematic plan [1], (b) sketch of Musmeci project by Sigel [8] 
The structure is designed to be entirely built of reinforced concrete, in which the parabolic webs are self-bearing shells, and the ribs are arches with flexural stiffness that convey loads to the corner abutment pillars [1].

According to Musmeci's idea, the direct correlation between the static functionality and the architectural form of the structure is further represented by the trend of the stresses in the ribs. The ribs are subjected to vertical loads that vary linearly from the crown to the abutment, and to constant horizontal thrusts. Therefore, Musmeci deduced that, though the parabolic centreline of the ribs does not correspond to the funicular line of the vertical loads, since these are not constant, the presence of horizontal thrusts brings the funicular line very close to the parabolic one, thus reducing the maximum eccentricity to few centimetres [1].

\subsection{The Musmeci structural model}

The Musmeci structural model [1] is here discussed referring to the central surface of a single bay of the tripartite vault. A Cartesian reference system $\{0 ; x, y, z\}$ with origin $O$ at the crown and positive $z$-axis downwards is introduced, as depicted in Figure 2.
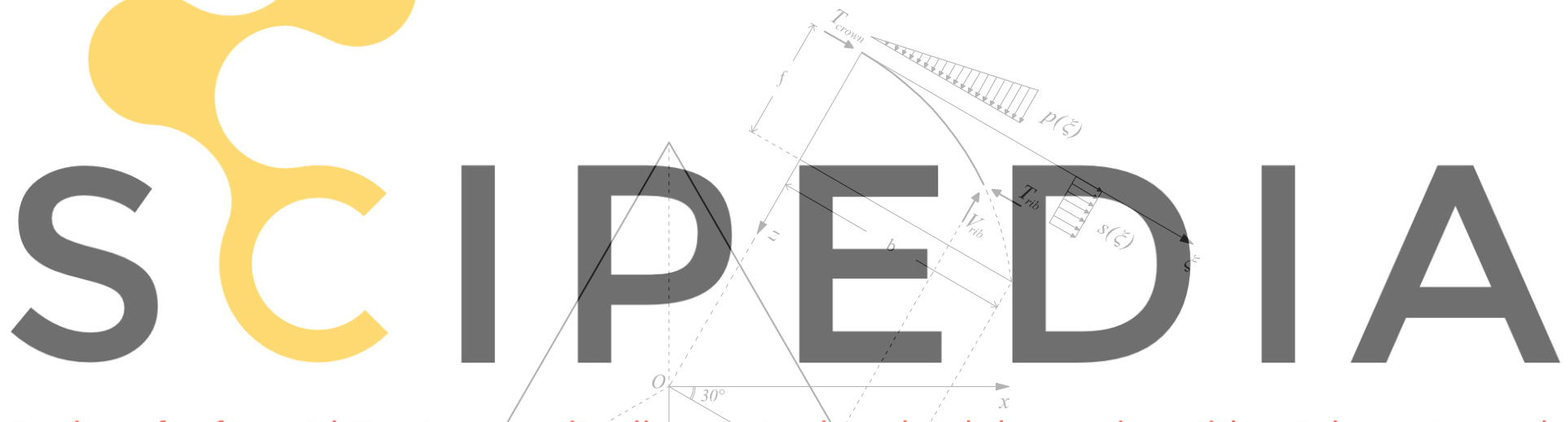

Register for free at https//www.scipedia.com to download the version without the watermark

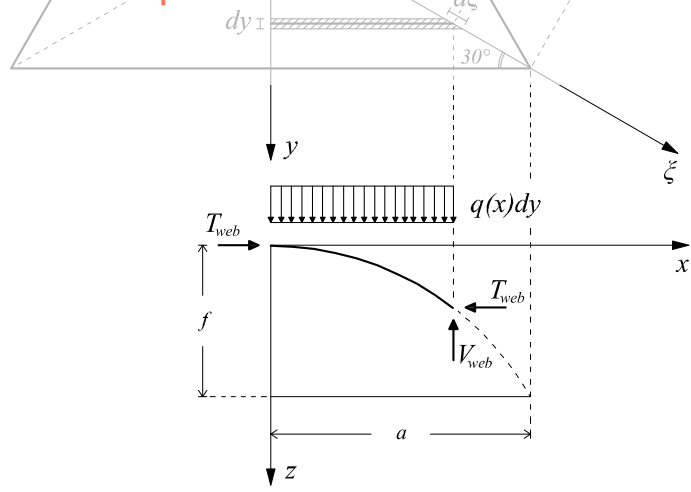

Figure 2: Scheme of the Musmeci structural model

Dividing the webs into arches with infinitesimal width $d y$, the equation of the arches can be written as:

$$
z(x)=\frac{f}{a^{2}} x^{2},
$$


and if $q$ is the vertical load per unit surface in horizontal projection, the thrust is equal to:

$$
T_{w e b}=\frac{q a^{2}}{2 f} d y .
$$

Rotating by $30^{\circ}$ about the $z$-axis the coordinate system and choosing $\xi$ as new abscissa, the equation of the ribs is:

$$
z(\xi)=\frac{f}{b^{2}} \xi^{2}=\frac{3 f}{4 a^{2}} \xi^{2}
$$

since the rise is again $f$ and the half span is $b=a / \cos \left(30^{\circ}\right)=2 a / \sqrt{3}=7.22 \mathrm{~m}$.

The vertical load acting on the ribs per unit length in horizontal projection is:

$$
p(\xi)=2 q x d y=\frac{\sqrt{3}}{2} q \xi d \xi
$$

and it is accompanied by a horizontal component resulting from the thrust $T_{\text {web }}$ of two webs:

$$
s(\xi)=2 T_{\text {web }} \cos \left(30^{\circ}\right)=\frac{\sqrt{3}}{4} \frac{q a^{2}}{f} d \xi=\frac{3 \sqrt{3}}{16} \frac{q b^{2}}{f} d \xi
$$

Therefore while $p$ varies linearly with $\xi, s$ is constant.

Under these loads, the rib is subjected to bending stresses since its profile does not coincide with their funicular line. In addition, as explained in [1] by Musmeci: "Since the flexural stiffness of rib is practically negligible at the abutment, where the section is theoretically

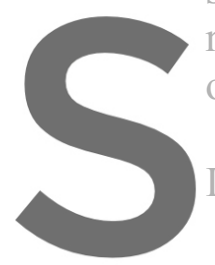
reduced to a point, and of the rib can be considera Let $V$ and $T$ respectively In particular, $V$ is equal to:
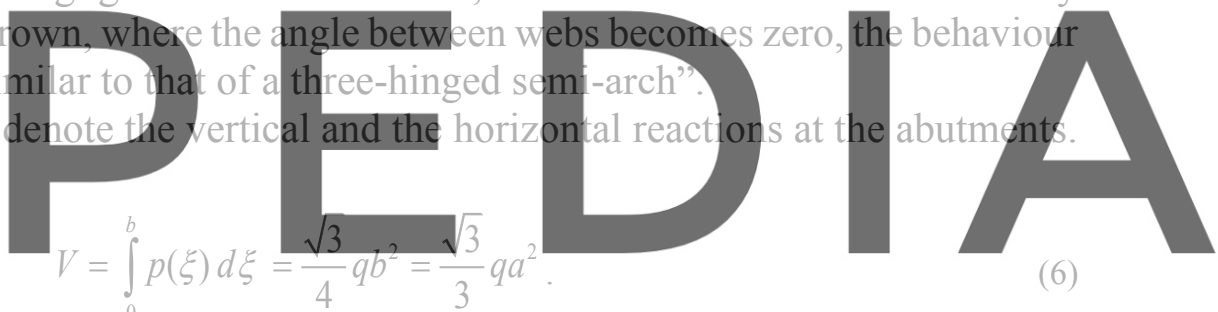

(6)

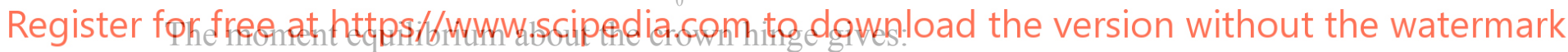

$$
V b-\int_{0}^{b} p(\xi) \xi d \xi+\int_{0}^{b} s(\xi) z(\xi) d \xi-T f=0,
$$

which allows immediately to calculate the thrust $T$ :

$$
T=\frac{7 \sqrt{3}}{48} \frac{q b^{3}}{f}=\frac{7}{18} \frac{q a^{3}}{f} .
$$

At the crown the thrust value is:

$$
T_{\text {crown }}=T-\int_{0}^{b} s(\xi) d \xi=T-s(\xi) b=-\frac{\sqrt{3}}{24} \frac{q b^{3}}{f}=-\frac{q a^{3}}{9 f},
$$

and the negative sign indicates the presence of traction in the rib. In particular, since the thrust varies linearly along the rib, it will be zero at $\xi=2 / 9 b=1.60 \mathrm{~m}$. As consequence of traction, a reinforcement is designed as shown in Figure 3 [1].

In the Musmeci project, the vault thickness ranging from 12 to $8 \mathrm{~cm}$, tapering in key, and the webs are connected along the external side by 15 to $22 \mathrm{~cm}$ thick edge reinforced curbs, with an almost rectangular section. The ribs have a triangular section with height of $28 \mathrm{~cm}$ and are 
reinforced in the same way as the curbs. It can be noticed that the longitudinal and transversal bars arrangement along the ribs underlines their structural function as curvilinear beams in the Musmeci model.

(a)

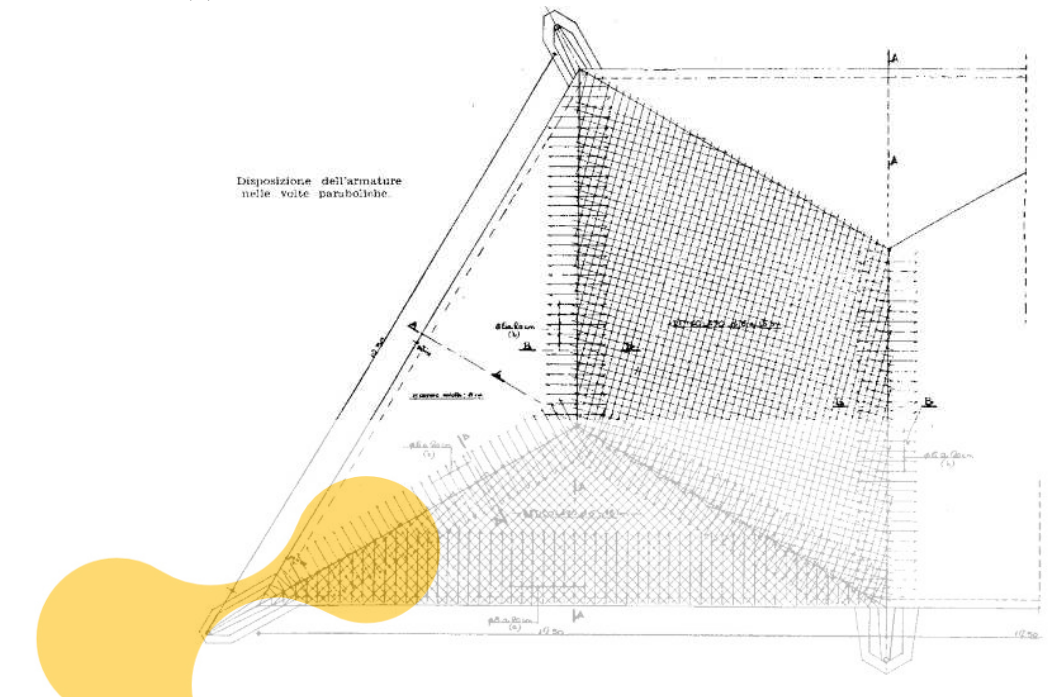

(b)

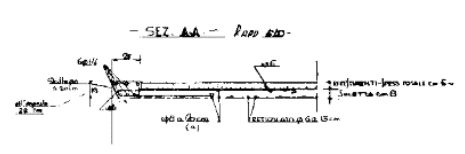

(c)

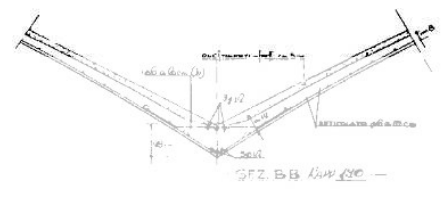

Figure 3: Reinforcement detailing of parabolic vaults [1]: (a) plan view, (b) cross-section of curbs and (c) cross-

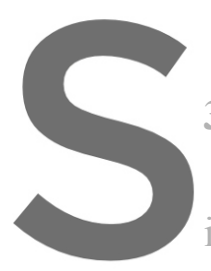

Tre

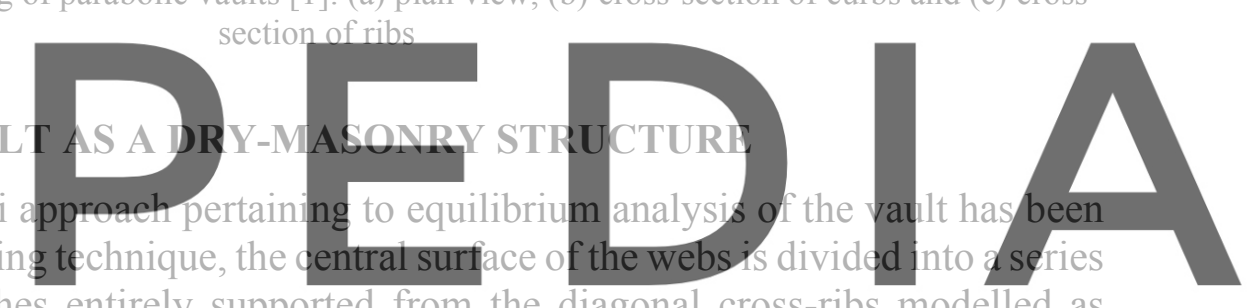

of independent parallel arches entirely supported from the diagonal cross-ribs modelled as

Register for tree athitths/\%ww. w scipedia.com to download the version without the watermark

successively developed by Heyman $[3,4]$ to clarify the path of the forces in masonry domes and

masonry cross vaults and to evaluate the thrust value. Furthermore, another link between the presented reinforced concrete vault and classical masonry vaults is pointed out by Musmeci's introduction of the edge curbs. Indeed, the stiffen of external web arches along the free sides of the structure recalls the formeret arches in a quadripartite Gothic masonry vault.

In this context, the aim of the present work is to propose in a novel prospective the unbuilt Musmeci vault, reconsidered as a dry-masonry structure. In order to respect the original shape of the vault, the unique design parameter is the thickness $t$. In detail, the starting point is the discretization of the vault through the research on the appropriate stereotomy of blocks.

The main assumptions are: (i) the webs are coursed in the 'French' way [3] and in the structural model they are divided into a series of parallel arches of variable rise, span and width but with uniform thickness $t$; (ii) the ideal ribs, generated by the intersection at $120^{\circ}$ in plan of two parabolic webs, are modelled as parabolic arches with variable thickness and vanishing width (in particular, at the crown the ribs thickness is equal to $t$ ); (iii) all arches are divided into a finite number of voussoirs with joints normal to the curvilinear axis of the web arches (in order to minimize the shear components along the interfaces). Conversely, the ideal voussoirs 
of the ribs do not have interface normal to their axes, being generated by the projection of the joints of the web longitudinal courses on diagonal planes of the ribs. Finally, the design voussoir size is investigated in the range between $2.5^{\circ}$ and $5^{\circ}$. Two examples of the geometry discretization of the Musmeci vault are shown in Figure 4. Taking advantage of the geometric symmetry, only one-sixth portion of the vault is modelled.

(a)

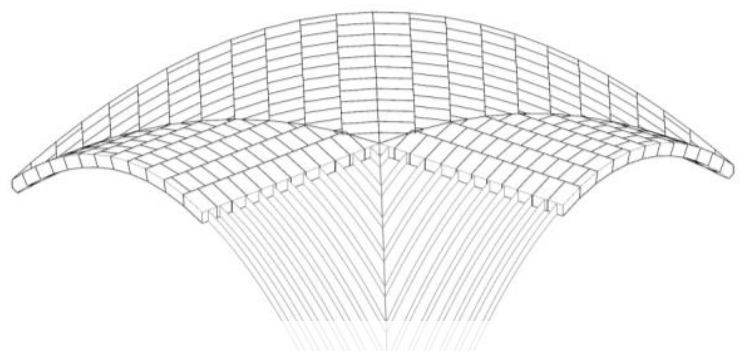

(b)

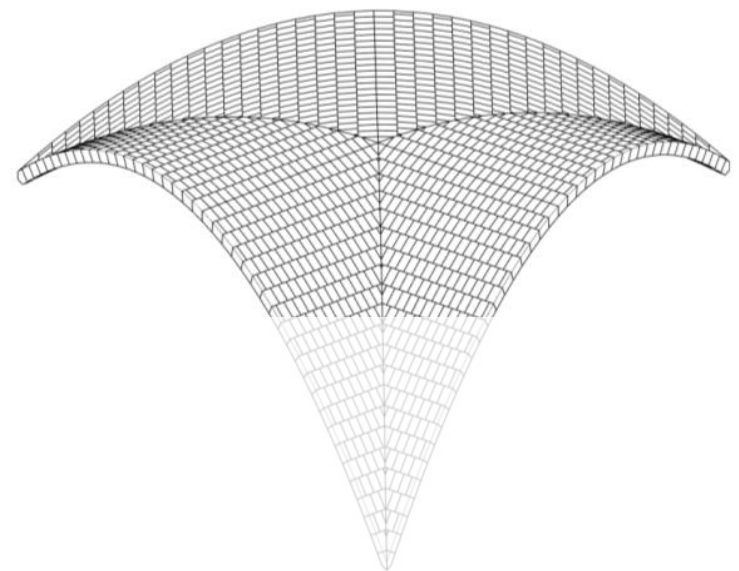

Figure 4: Geometry discretization of Musmeci vault, reconsidered as a dry-masonry structure, with thickness equal to $t=24 \mathrm{~cm}$ and: (a) voussoir size equals to $5^{\circ}$ (and the relevant sliced model considered) and (b) to $2.5^{\circ}$
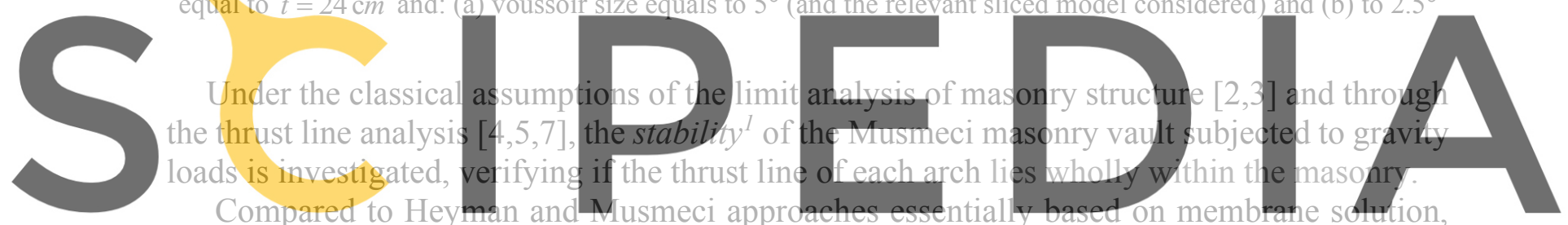

with the present approach it is possible to take full advantage of the actual thickness of the vault

Register forcfireeta屯 hotfpesdigw ww.scipedia.com to download the version without the watermark

First, in the spirit of the minimum structural design criteria, the minimum thickness of the vault is investigated and discussed. Furthermore, adopting the procedure initially proposed for circular masonry arches in $[5,7,11]$ and then considered for square cross vaults in [10], the vault capacity on spreading supports is assessed in terms of ultimate displacements. Since the behaviour of the masonry vault undergoing settlements is deeply connected to the behaviour of its ribs, the case of the parabolic arch is first analysed in Section 4.

\section{MASONRY PARABOLIC ARCH ON SPREADING SUPPORTS}

This section is devoted to evaluating the behaviour of a masonry parabolic arch undergoing horizontal settlements at the springing. In Section 4.1, attention is focused on the adopted methodology and on compatible mechanisms that can lead the arch to a collapse configuration. In Section 4.2, the least thickness and the minimum thrust of the parabolic arches, generated by the discretization of the Musmeci vault, are analysed for different values of the thickness ratio t/a. Finally, in Section 4.3 the spread limits and the relative thrust at the collapse are discussed and reported. All results have been achieved by means of an inhouse MATLAB ${ }^{\circ}$ code.

\footnotetext{
${ }^{1}$ As defined by the Heyman "safe" theorem [2-4].
} 


\subsection{Static approach and compatible mechanisms in deformed configuration}

The capacity of masonry arches to withstand the actions of the environment, such as differential foundation settlements or earthquakes, lies precisely in the possibility of opening cracks [2]. The cracks are therefore not dangerous but a natural consequence of the aptitude of a masonry structure to absorb the external actions.

In this framework, under the well-known assumptions of standard limit analysis theory adopted for masonry structure [2], the equilibrium approach [5] has been applied to investigate the behaviour of a parabolic arch subjected to self-weight and horizontal displacements at the abutments. In particular, as soon as the centring is removed, the thrust is activated and the supports, being not fixed, undergo a slight yielding. According to [2,7], the small movement causes the formation of three hinges in the arch, at the extrados near the crown, and at the intrados near the haunches, and the thrust at the abutments of the arch is the lowest of all the statically admissible ones. As discussed in [9], the calculation of the minimum thrust $T_{0}$ in undeformed configuration can be interpreted as the following linear optimization problem:

$\min f^{t} x$ s.t. $A x \leq b$,

where $x$ is the $2 \times 1$ vector collecting the hyperstatic reactions (chosen as the moment and the thrust at the crown section) and $f$ is the $2 \times 1$ equilibrium operator such that $f^{t} x$ gives the thrust at the springing section. Under the assumption of infinite compressive strength of the material, the inequality constraint enforces the pressure points to lie inside the $M$ joint sections of the

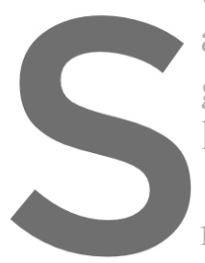
arch. In detail, $A$ [resp., $b]$ is a $2 M \times 2$ matrix
gives the moments of the hyperstatic reactions
loads] with respect to the intrados and extrados
Starting from this configuration and increa
rigid body kinematics $[5]$. Assuming a synn
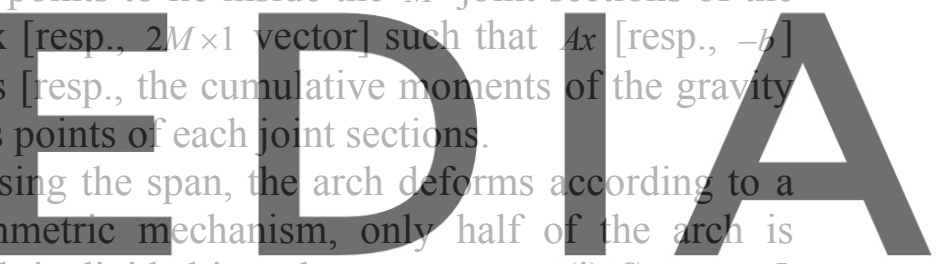
considered. In particular as in [9], the half arch is divided into three segments: (i) Segment I,

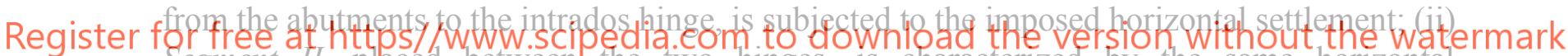
Segment II, placed between the two hinges, is characterized by the same horizontal displacement and by a rotation around the intrados hinge; (iii) Segment III, from the extrados hinge to the crown section, is only characterized by a vertical translation. As for semi-circular arches and elliptical arches [5,9], during the displacement of the supports and the consequent kinematic motion, the intrados and extrados hinges might jump in different locations from the starting ones.

A parametric survey is carried out in order to understand the influence of the main geometric parameters on the global response of a parabolic arch. In particular, the external web arch generated by the geometry discretization described in Section 3 and the diagonal arch are considered. In this regard, it should be noticed that the diagonal arch has variable thickness, at the crown equal to $t$ and gradually increasing towards to the support, and a value of rise over span ratio $f / a$ equal to 0.506 (also the diagonal semi-span will be indicated from here on with a) while for the external web arch $f / a$ is equal to 0.584 .

Finally, the analyses are performed for different sizes of the voussoirs, but, for space reasons, only the results relating to the case of voussoirs size equal to $2.5^{\circ}$ (i.e. voussoir length equals to about $31 \mathrm{~cm}$ ) are reported in the next sections. 


\subsection{Minimum thickness and minimum thrust of parabolic arch}

The minimum thrust $T_{0}$, normalized by the total arch weight $W$, versus the thickness ratio $t / a$ evaluated at the crown section, is plotted in Figure 5 for different values of $t / a$. The curves start at the least possible thickness. In this situation, the minimum thrust is equal to the maximum one, and the arch reaches a state of limit equilibrium. In particular, the parabolic arches exhibit a behaviour similar to that of flying buttress in the passive state, described in the case of uniform thickness in [2,3] and with curved intrados in [11]. Indeed, the thrust line passes through intrados at the crown and at the springing, touching extrados just below the midpoint, as depicted in the red box (diagonal arch) and yellow one (external web arch) of Figure 5.

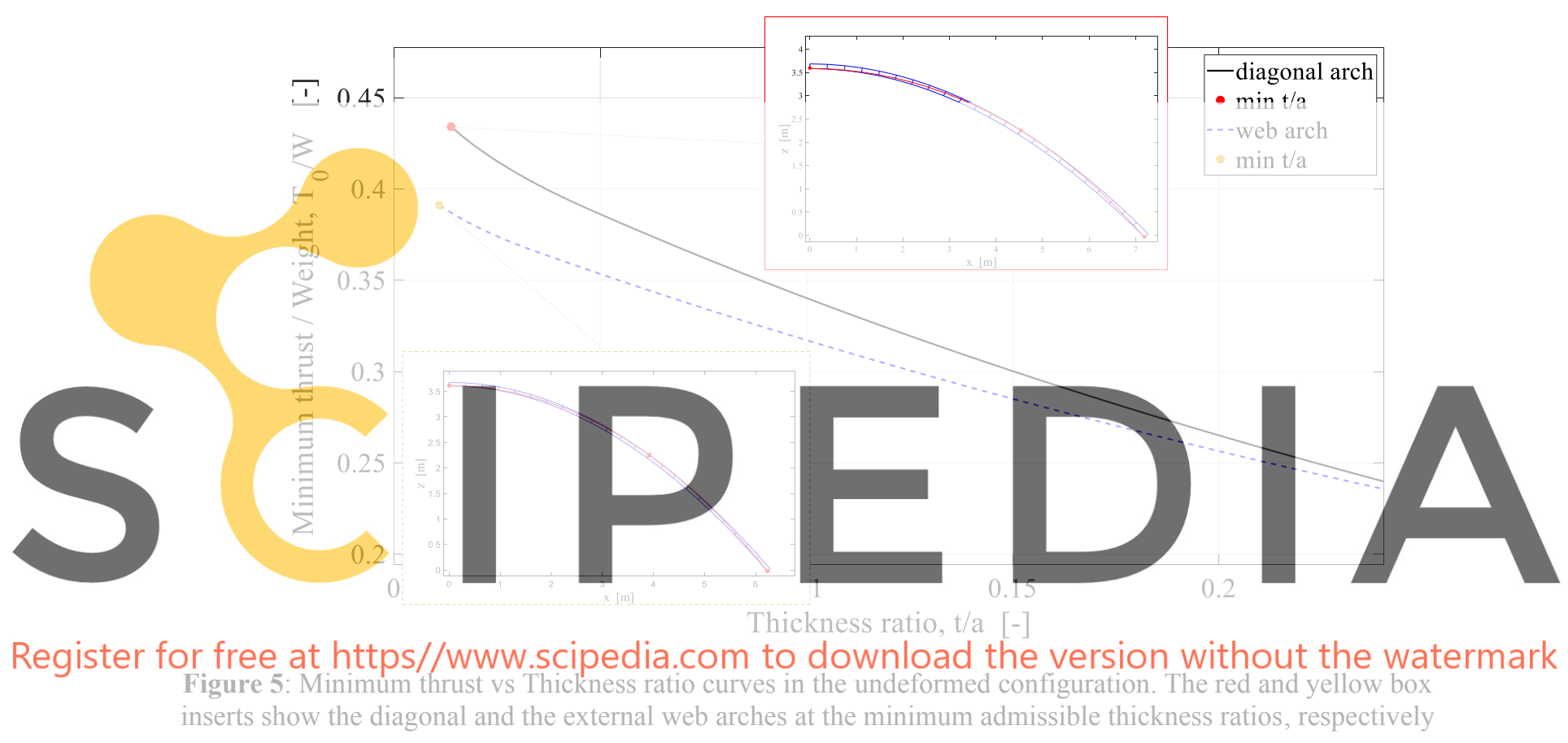

For the diagonal and external web arches, the minimum thickness values are $t / a=0.014$ and 0.011 , i.e. $t=6.88 \mathrm{~cm}$ and $10.10 \mathrm{~cm}$, with values of $T_{0} / W=0.434$ and 0.391 , respectively. Despite the variable thickness, as a consequence of the significant lower value of $f / a$ ratio, the diagonal arch has greater values of $T_{0} / W$ than the web arch, for each value of $t / a$. Nevertheless, the smallest admissible value of $t / a$ is attained by the web arch. This result is related to the effect of voussoir stereotomy on the shape of the thrust line [12]. In fact, the discretization through normal cuts of the web arches implies their possibility of accommodating a larger set of thrust lines within their thickness ${ }^{2}$.

Moreover, it is worth noticing that the minimum thickness of the web arch is about an order of magnitude lower than the minimum thickness of the elliptical arch with same $f / a$ and $t / a$

\footnotetext{
${ }^{2}$ In this regard, the minimum thickness ratio $t / a$ of the same diagonal parabolic arch, with joints normal to its axis, is 0.0075 .
} 
ratios [12], since the parabolic profile is closer to the catenary curve.

Finally, as the thickness ratio increases, both curves exhibit a decrease of values of minimum thrust with a trend similar to the one observed for circular arches [10] and elliptical ones [12].

\subsection{Collapse displacement and relative thrust of parabolic arch}

With the aim to assess the behaviour of the parabolic arch undergoing horizontal displacement at abutments, the cases pertaining to the diagonal and the external web arches are analysed using the limit analysis procedure and their capacity is evaluated in terms of collapse displacement and relative thrust.

As originally proposed for the circular arches in [5], the thrust increase, defined as the ratio of the thrust at collapse displacement $T_{\text {coll }}$ to the minimum thrust $T_{0}$, is plotted versus the thickness ratio $t / a$ in Figure 6a. A practical limit is introduced, i.e. the behaviour of the arches is not investigated when the thrust becomes greater than five times the minimum thrust $[9,10]$.
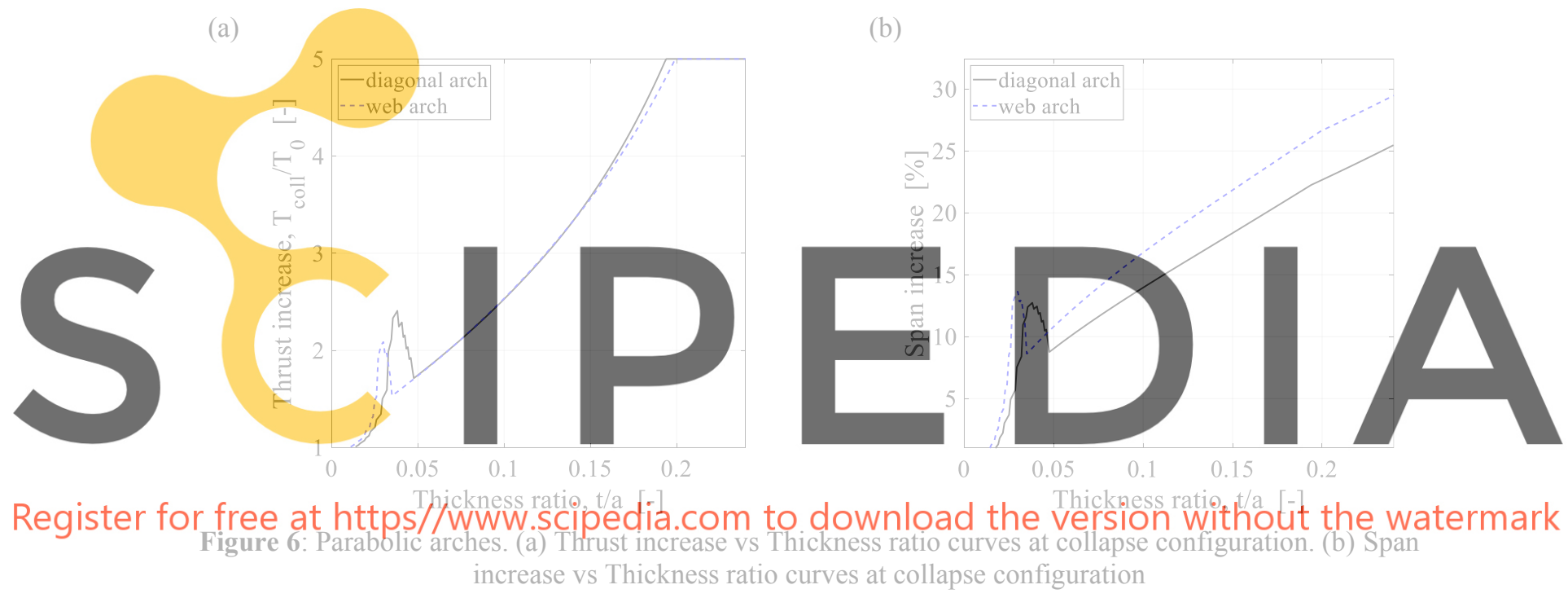

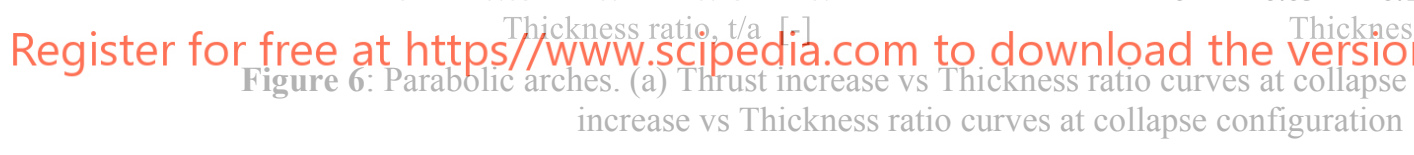

The governing failure mechanisms for the parabolic arches on spreading supports can be classified into three types, corresponding to three regions of the curves in Figure 6.

In the first region, for low thickness ratio values until the first peak, both arches fail due to a five-hinge mechanism described in Section 4.2. In particular, the extrados hinge remains located in the same position of the corresponding case of minimum thrust, whereas moving the supports apart the line of pressure touches the intrados at the crown, activating the mechanism.

Increasing the thickness, the location of the initial extrados hinge moves toward the crown of the arch and for small increments of $t / a$ ratio there are large increases in both thrust and corresponding collapse displacement. Unlike the case of flying buttresses described in [11], the formation of the intrados hinge at the crown leads to the failure of the arch, preventing the achieving of the snap-through mechanism.

The second region is within the $t / a$ range of $0.038-0.048$ and $0.030-0.035$, for the diagonal and the web arches, respectively. In details, starting from local peaks, the collapse mechanism changes and jumps of both extrados and intrados hinges occur, modifying the geometry of the 
arch and overcoming the static admissibility. Indeed, as presented in [5,9,10], if for a fixed value of the support displacement the line of pressure becomes tangential to two adjacent joints, the gravitational loads cause the closure of the lower hinge and the opening of the upper one. These jumps bring to different collapse configurations, producing discontinuities in the thrust increase clearly visible in the post-peak zone of solid curve of Figure 6a, pertaining to the diagonal arch.

In the third region, the thickness is sufficiently large to accommodate a thrust line passing through the crown at the extrados and through the springing section at the intrados, even in the undeformed configuration. Moving the supports apart, the location of the extrados hinge remains fixed, the arch approaches a classical snap-through failure, but the jump of the intrados hinge causes the failure. The same mechanism has been found for depressed circular arches in $[5,10]$, with a shape of curves very similar to those shown in Figure 6a, for a comparable range of $t / a$. Finally, for $t / a$ values approximately greater than 0.20 , the practical limit is achieved.

The corresponding values of the span increase, defined as the percentage of the ratio of the displacement at collapse to the initial clear span, are depicted in Figure 6b. The trend of the curves is in accordance with the different collapse mechanisms previously described. In particular, it can be noticed that in the third region the slope of the curve is almost linear with a slight slope decrease at values of $t / a$ corresponding to attainments of the practical limit of the thrust increase.

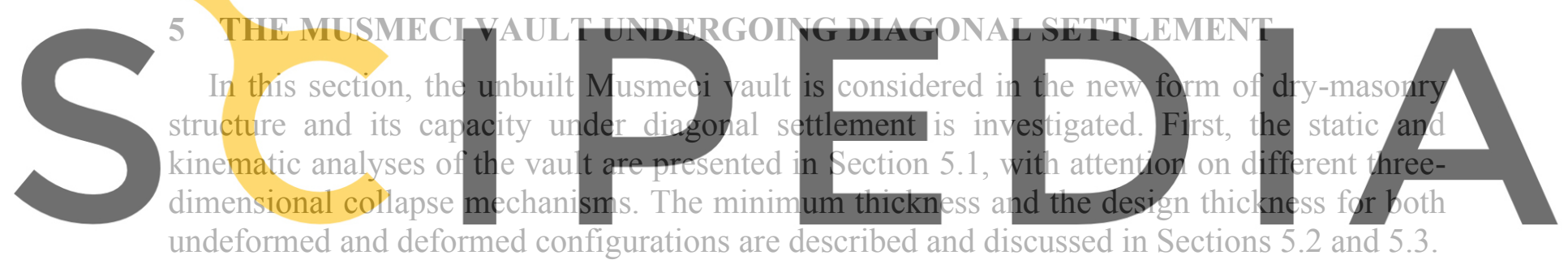

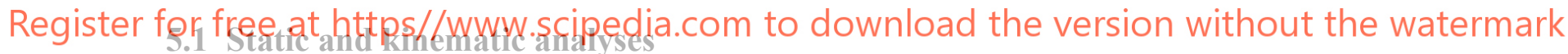

The static analysis of vaults under dead loads is a classical problem in masonry structures, mainly addressed to their conservation and maintenance but recently also as a new method for the design exploration of compression-only structures [14].

Generally, once the support structures have been removed, the activation of the thrust at the supports entails a small settlement, with a slight widening of the ribs and of the webs themselves. Small natural cracks occur, and the minimum thrust state of the vault is activated.

According to [7], the behaviour of the cracked vault can be analysed through the sliced model, under the assumption of rigid no-tension masonry model. As presented in [10], a linear optimization problem, similar to the one in Eq. (10), is solved for the hyperstatic reactions (i.e., the thrust and the moment at the crown of diagonal arch and of each web arch), enforcing the pressure points to lie inside all vault joints. An example of the results of the equilibrium analysis and the corresponding thrust networks is depicted in Figure 7.

Starting from the undeformed configuration, the weight of the vault is divided into the web arches which transmit their horizontal and vertical reactions in the rib plane. The thrusts of the web arches are combined with those of the adjacent web along the diagonal plane and imply a variable horizontal load on the rib where the minimum thrust at the abutment is sought. 
Therefore, the initial statically indeterminate problem can be uniquely solved, and a unique hinges pattern in the arches is determined. According to the $f / a$ ratio of each arch and the vault thickness $t$ analysed, a different couple of extrados and intrados hinges can occur in each arch. Since the original geometry of Musmeci vault has low $f / a$ ratio, the thrust lines of each arch passes through the intrados at the springing.

Enforcing a diagonal horizontal displacement at the abutments, the deformed configuration of the vault is analysed. In particular, the deformation of the ribs as a set of rigid bodies (i.e. the three segments introduced in Section 4.1) causes the aperture of cracks that completely split the overlying web. It should be noticed that these mechanisms are able to depict the cracking patterns typically detected for these type of faults [3,7].

As a consequence of the geometric discretization of the vault, each web arch rests on one voussoir of the ribs and undergoes a different possible settlement, according to the pertinent rib segments.

(a)

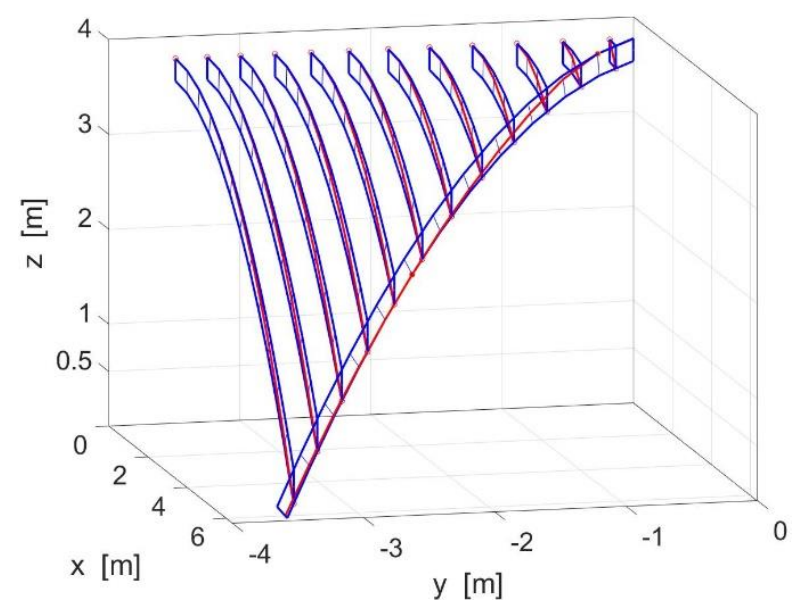

(b)

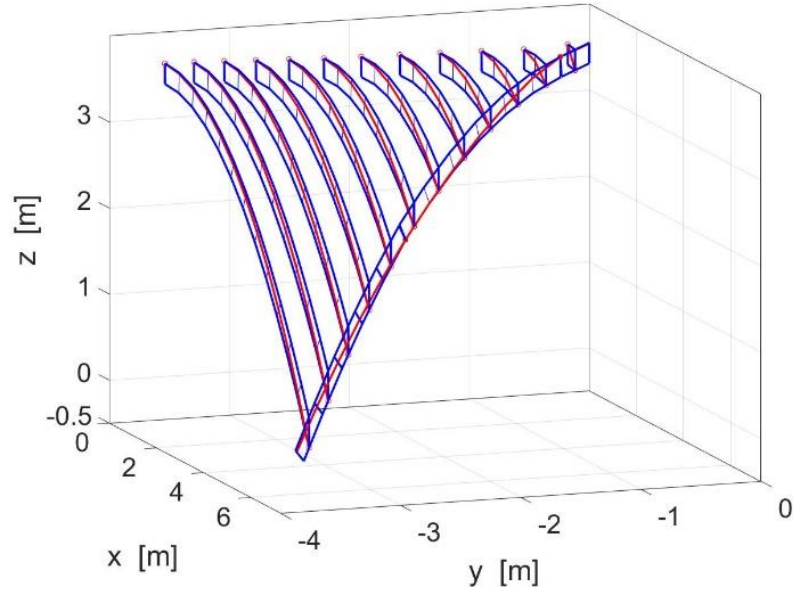

Figure 7: Musmeci vault. Thrust analysis at (a) undeformed configuration and (b) collapse configuration. One sixth of the vault is modelled, with voussoir size of $5^{\circ}$ and thickness equal to $t=24 \mathrm{~cm}$, is here considered

Therefore, as presented in [9], the arch webs can be also divided into three classes. Arches of Class I, pertinent to the Segments I of the ribs, are in the outer zone of the web and are subject to a horizontal settlement of their springing. Arches of Class II, located between the two hinges of the rib, are in contact with the rib only through their intrados hinge and are subject to both vertical and horizontal settlements. Finally, arches of Class III, located in the inner zone of the web near the vault key, undergo a vertical translation. Furthermore, in order to have an admissible kinematic mechanism, the Class I and II arches are dragged by a rigid translation orthogonal to their plane. An example of the results of the analysis at collapse displacement is shown in Figure $7 b$.

\subsection{Minimum thickness and minimum thrust of Musmeci vault}

In Figure 8, the minimum thrust of the cross vault, normalized with respect to its total weight 
is presented for different values of the thickness ratio, $t / a$.

The trend of the curve is similar to the previous ones shown for the case of the parabolic arch. The curve starts with the minimum thickness ratio (red dot), corresponding to a limit equilibrium state of the vault. In this situation, the mechanism is achieved when the pressure line passes through the extrados at the springing of the diagonal rib, with an incipient five-hinge symmetric mechanism of the ribs. Moreover, it can be noticed that the design thickness ratio t/a, whose choice will be discussed later, is marked with the green square on the curve. In particular, the values of minimum thickness and the design thickness are $t / a=0.019$ and 0.033 , i.e. $t=14 \mathrm{~cm}$ and $24 \mathrm{~cm}$, with values of $T_{0} / W=0.372$ and 0.350 , respectively.

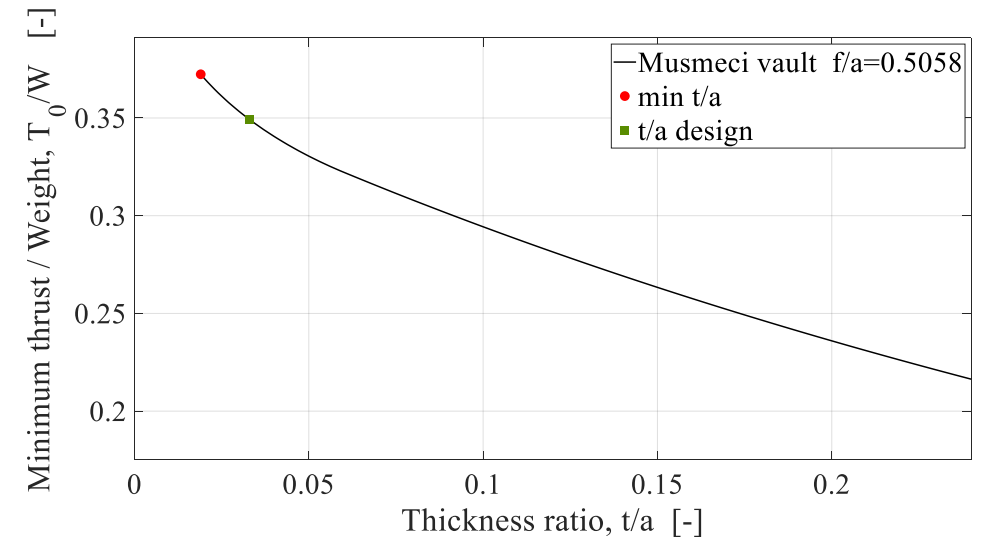

Figure 8: Musmeci vault. Minimum thrust vs Thickness ratio curves in the undeformed configuration

\subsection{Displacement capacity of Musmeci vault}

In Figure 9a the thrust increase is shown versus the thickness ratio $t / a$. As before, the curve is truncated when the thrust, at the springing section of the rib, achieves the practical limit.

The failure mechanisms of the cross vault are closely related to the behaviour of their ribs. In particular, for low values of the thickness, the vault collapse is attained due to a five-hinge mechanism in the ribs. Unlike the parabolic arch case (see Section 4.2), it should be noticed that in this case the pressure line touches the intrados towards the haunches and the extrados near the crown and at the supports of the ribs (an example is depicted in Figure 6b). This change in their behaviour is due to the action of the thrusts transmitted by the web arches to the rib.

Increasing the thickness ratio, the initial intrados hinge of the ribs moves from the haunch toward the abutment, involving not only a change in the geometry of the ribs but also a change of the Class of the web arches (see Section 5.1). Moreover, these significant changes in the initial geometry of the vault lead to discontinuities in the values of the increase in thrust, bringing also to a different collapse mechanism pertaining to jumps of the intrados or extrados hinges.

From the value of $t / a$ equal to 0.084 onwards, the initial intrados hinge of the rib remains localized on the springing and the collapse mechanism of the vault approaching to snap-through failure and the practical limit is rapidly achieved.

In Figure $9 \mathrm{~b}$ the relevant span increase at collapse configuration is reported. The high 
displacement capacity of the vault to adapt to horizontal settlements is highlighted by the values of the span increase which grow rapidly up to over $30 \%$. The trend of the curves and the collapse mechanisms are similar than the ones found in [9] for rounded cross vault.

Finally, considering that the original Musmeci structure was designed as a cover for a rural market, without underlying structures, it was chosen as the design thickness of the vault $t=24 \mathrm{~cm}$, with a geometric factor of safety [2] equal to 1.71 , accordingly with the minimum structural design criteria of the author. The design size of voussoirs is chosen equal to $2.5^{\circ}$ as a value near to the standard dimensions of voussoirs used in practice.

An example of the final appearance of the Musmeci vault reconsidered as a dry-masonry structure is depicted in Figure $4 \mathrm{~b}$.

(a)

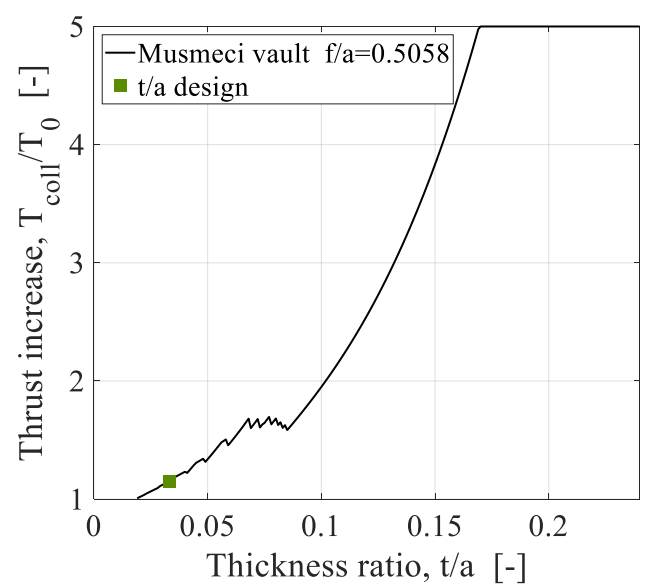

(b)

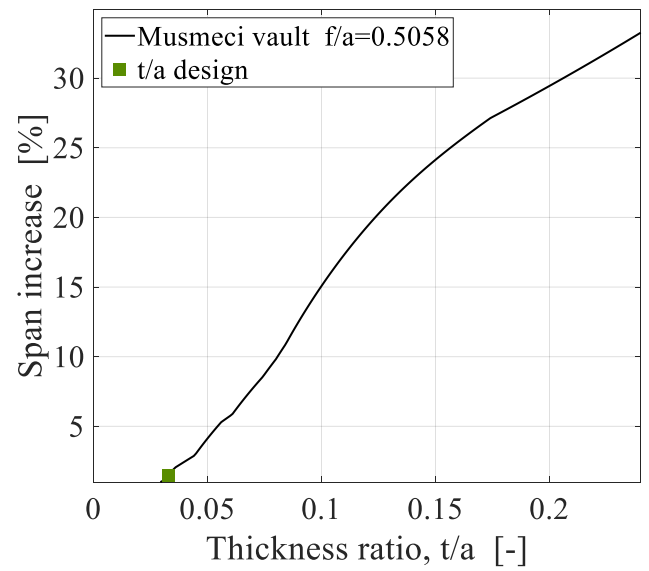

Figure 9: Musmeci vault. (a) Thrust increase vs Thickness ratio curves at collapse configuration. (b) Span increase vs Thickness ratio curves at collapse configuration

\section{CONCLUSIONS}

The unbuilt parabolic Musmeci cross vault has been proposed in the novel perspective of dry-masonry structure. In order to respect the original shape, the thickness of the vault has been considered as the unique design parameter. First, the geometry discretization of the reinvented vault and the main assumptions of the proposed model have been discussed. Second, the minimum thrust of the vault and its displacement capacity undergoing horizontal settlements have been investigated for different thickness ratio values. The Musmeci vault shows a low value of the minimum thickness and significant span increase at collapse, comparable to the parabolic arch constituting its diagonal rib. In particular, for low thickness ratio values, the failure is due to a five-hinge mechanism. Increasing the thickness, the collapse mechanism approaches a snap-through failure, the thrust increases rapidly and several hinge movements occur. The jump of the hinges involves a sudden change in the deformed configuration that, in some cases, may no longer be statically admissible, consequently leading to a premature collapse of the system. Compared to the original vault of Musmeci, designed to be entirely built in reinforced concrete, the proposed model provides an estimate of a reasonable design thickness of the vault, showing the possibility to bring a new life to this historical and elegant structure, as a dry-masonry vault. 


\section{REFERENCES}

[1] Musmeci, S. and Vaccaro, G. Copertura a volte paraboliche per un mercato rurale. L'Ingegnere (1954) 5:487-490.

[2] Heyman, J. The stone skeleton. Int. J. Solids Struct. (1966) 2:249-279.

[3] Heyman, J. The stone skeleton: structural engineering of masonry architecture. Cambridge University Press, (1997).

[4] Huerta, S. Mechanics of masonry vaults: The equilibrium approach. In: P.B. Lourenco et al. (Eds.): Historic Constructions, University of Minho (2001), pp. 47-69.

[5] Ochsendorf, J. A. Collapse of masonry structures. PhD thesis, Department of Engineering, Cambridge University, Cambridge, (2002).

[6] Nodargi, N.A., Intrigila, C. and Bisegna, P. A variational-based fixed-point algorithm for the limit analysis of dry-masonry block structures with non-associative Coulomb friction. Int. J. Mech. Sci. (2019) 161105078.

[7] Como, M. Statics of historic masonry constructions. Springer, Vol. I, (2013), Vol. II, (2016).

[8] Siegel, C. Strukturformen der modernen Architektur. Munich, (1961)

[9] Intrigila, C., Nodargi, N.A. and Bisegna, P. Square cross vaults on spreading supports. In: R. Aguilar et al. (Eds.): Structural Analysis of Historical Constructions, RILEM Bookseries 18 (2019), pp. 1045-1053.

[10] Coccia, S., Di Carlo, F. and Rinaldi, Z. Collapse displacements for a mechanism of spreading-induced supports in a masonry arch. Int. J. Adv. Struct. Eng. (2015) 7:307-320.

[11] Nikolinakou, M. K., Tallon, A. J., and Ochsendorf, J. A. Structure and form of early Gothic flying buttresses. Revue européenne de génie civil (2005) 9:1191-1217.

[12] Makris, N. and Alexakis, H. The effect of stereotomy on the shape of the thrust-line and the minimum thickness of semicircular masonry arches. Arch. Appl. Mech. (2013) 83:1511-1533.

[13] Alexakis, H. and Makris, N. Minimum thickness of elliptical masonry arches. Acta Mech. (2013) 224: 2977-2991.

[14] Block, P. and Ochsendorf, J.A. Thrust network analysis: a new methodology for threedimensional equilibrium. J. Int. Assoc. Shell Spat. Struct. (2007) 48:67-173. 